\title{
Potassium channels in C. elegans ${ }^{*}$
}

\author{
L. Salkoff ${ }^{1}, 2, \S$, A.D. Wei ${ }^{1}$, B. Baban ${ }^{1}$, A. Butler ${ }^{1}$, G. Fawcett ${ }^{1}$, \\ G. Ferreira ${ }^{1}$, C.M. Santi ${ }^{1}$
}

${ }^{1}$ Department of Anatomy and Neurobiology and

${ }^{2}$ Department of Genetics, Washington University School of Medicine, Saint Louis MO, 63110 USA

\section{Table of Contents}

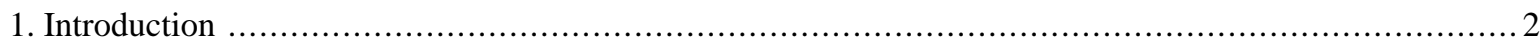

1.1. Many potassium channels in $C$. elegans have close mammalian orthologues .......................2

1.2. Genetic screens for abnormal behavioral phenotypes reveal potassium channel mutants ............ 3

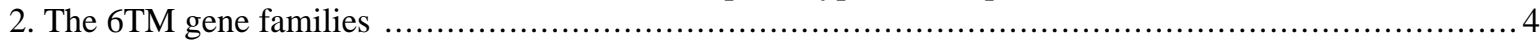

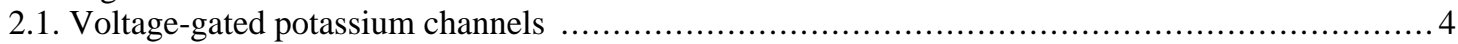

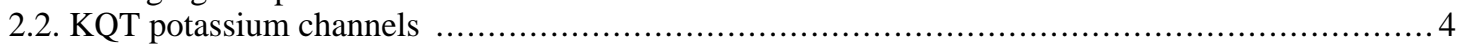

2.3. Eag-like potassium channels ................................................................. 5

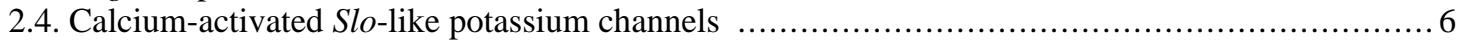

2.5. Cyclic nucleotide-gated cation channels ...................................................... 7

2.6. SK Small conductance, voltage insensitive calcium-activated potassium channels .................. 7

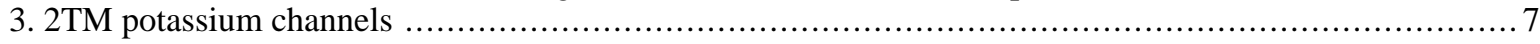

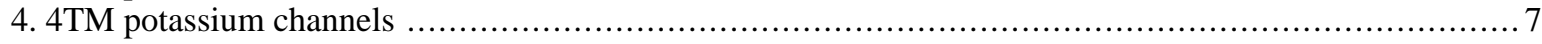

4.1. Evolution may tune the excitability of individual neurons using $4 \mathrm{TM}$ channels $\ldots \ldots \ldots \ldots \ldots \ldots \ldots . \ldots$

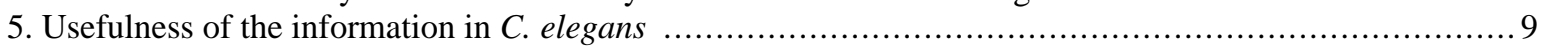

5.1. C. elegans can be a useful system to dissect apart the components of cell excitability using its

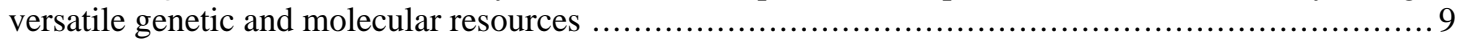

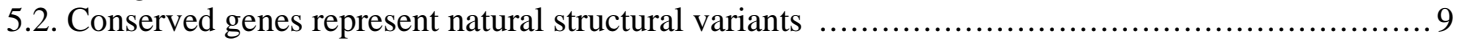

5.3. C. elegans could be a useful model system for studying genetic disease which involves ion channels

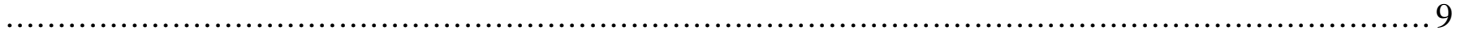

5.4. The study of ion channel functional properties in C. elegans has revealed unusual properties never

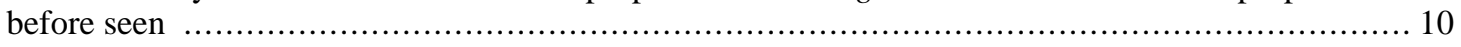

5.5. Mutant analysis in C. elegans has revealed gain-of-function mutants with possible therapeutic uses

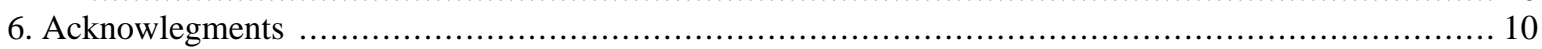

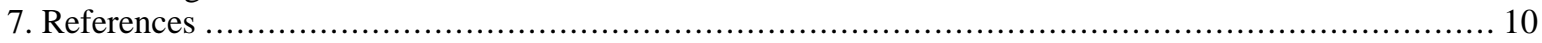

${ }^{*}$ Edited by Erik M. Jorgensen and Joshua M. Kaplan. Last revised December 19, 2005. Published December 30, 2005. This chapter should be cited as: Salkoff, et al. Potassium channels in C. elegans (December 30, 2005), WormBook, ed. The C. elegans Research Community, WormBook, doi/10.1895/wormbook.1.42.1, http://www.wormbook.org.

Copyright: $\odot 2005$ Salkoff et al., This is an open-access article distributed under the terms of the Creative Commons Attribution License, which permits unrestricted use, distribution, and reproduction in any medium, provided the original author and source are credited.

${ }^{\S}$ To whom correspondence should be addressed. E-mail: salkoffl@pcg.wustl.edu 
Abstract

Ion channels are the "transistors" (electronic switches) of the brain that generate and propagate electrical signals in the aqueous environment of the brain and nervous system. Potassium channels are particularly important because, not only do they shape dynamic electrical signaling, they also set the resting potentials of almost all animal cells. Without them, animal life as we know it would not exist, much less higher brain function.

Until the completion of the $C$. elegans genome sequencing project the size and diversity of the potassium channel extended gene family was not fully appreciated. Sequence data eventually revealed a total of approximately 70 genes encoding potassium channels out of the more than 19,000 genes in the genome. This seemed to be an unexpectedly high number of genes encoding potassium channels for an animal with a small nervous system of only 302 neurons. However, it became clear that potassium channels are expressed in all cell types, not only neurons, and that many cells express a complex palette of multiple potassium channels. All types of potassium channels found in $C$. elegans are conserved in mammals.

Clearly, C. elegans is "simple" only in having a limited number of cells dedicated to each organ system; it is certainly not simple with respect to its biochemistry and cell physiology.

\section{Introduction}

Potassium channels function in cellular tasks as ubiquitous as the setting of resting potentials (Katz, 1966), and as specific as defining the interspike intervals of endogenously beating neurons (Connor and Stevens, 1971). Not surprisingly, potassium channels are found in virtually all living cells including bacteria (Milkman, 1994; Saimi et al., 1999; Doyle et al., 1998; Jiang et al., 2002; Mackinnon and Doyle, 1997), yeast (Ketchum et al., 1995; Zhu et al., 1995), Paramecia (Jegla and Salkoff, 1995; Saimi et al., 1999), plants (Anderson et al., 1992; Sentenac et al., 1992 and Ketchum et al., 1996) and all animal cells, including electrically excitable tissues as well as inexcitable tissues, such as the liver (Pearson et al., 1999) and cells of the immune system (Cahalan and Chandy, 1997). Thus, potassium channels serve diverse functions such as shaping the dynamic electrical activity of neurons (Connor, 1980), modulating the immune response (Cahalan and Chandy, 1997) and controlling intestinal function (Koh et al., 1995). With the arrival of molecular biology and biochemical techniques, potassium currents in many cell types could be described not only electrophysiologically and pharmacologically, but also at the molecular level. Molecular techniques have also revealed a diversity far more extensive than was anticipated nearly two decades ago, when the first potassium channel gene was cloned from the Drosophila Shaker locus (Papazian et al., 1987; Kamb et al., 1987; Pongs et al., 1988).

\subsection{Many potassium channels in $C$. elegans have close mammalian orthologues}

Until the completion of the C. elegans genome sequencing project (C. elegans Sequencing Consortium, 1998) the size and diversity of the potassium channel extended gene family was not fully appreciated. Sequence data revealed a total of approximately 70 genes encoding potassium channels out of the more than 19,000 genes in the genome (Wei et al., 1996; Bargmann, 1998). This seemed to be an unexpectedly high number of genes encoding potassium channels for an animal with a small nervous system of only 302 neurons. However, it became clear that many potassium channels are expressed in all cell types, not only neurons (Salkoff et al., 1998) and that C. elegans is "simple" only in having a limited number of cells dedicated to each organ system; it is certainly not "simple" with respect to its biochemistry and physiology.

The genes encoding potassium channels can be divided into eight conserved families shown diagrammatically in Figure 1 (Wei et al., 1996). A universal feature of all potassium channels is a hyperconserved signature sequence (sometimes referred to as the "P" domain) that forms the potassium-selective filter of the pore (Heginbotham et al., 1992 , 1994). These eight families fall into one of three major subunit classes as defined by the number of "P" domains and transmembrane domains per subunit. Those possessing two transmembrane domains per subunit (2TM) bracketing a single P domain are categorized as "inward rectifier" channels. Those possessing four transmembrane domains (4TM) contain two P domains per subunit and represented a novel class of channels. Those possessing six transmembrane domains per subunit (6TM) with one P domain, include the voltage-gated potassium channel family. All potassium channels are symmetrical or semi-symmetrical structures and require four $\mathrm{P}$ domains per channel. Thus, four 2TM or four 6TM subunits are required to form a channel, while only two of the 4TM subunits are required to form a single channel of that class. Families, such as voltage-gated channels, can be further divided into multiple subfamilies (e.g., Shaker, Shab, Shaw and Shal; Figure 1). Within a single species there are commonly 
multiple members of each subfamily, such as Kv1.1 through Kv1.8, which represent eight mammalian homologues of the Shaker subfamily.

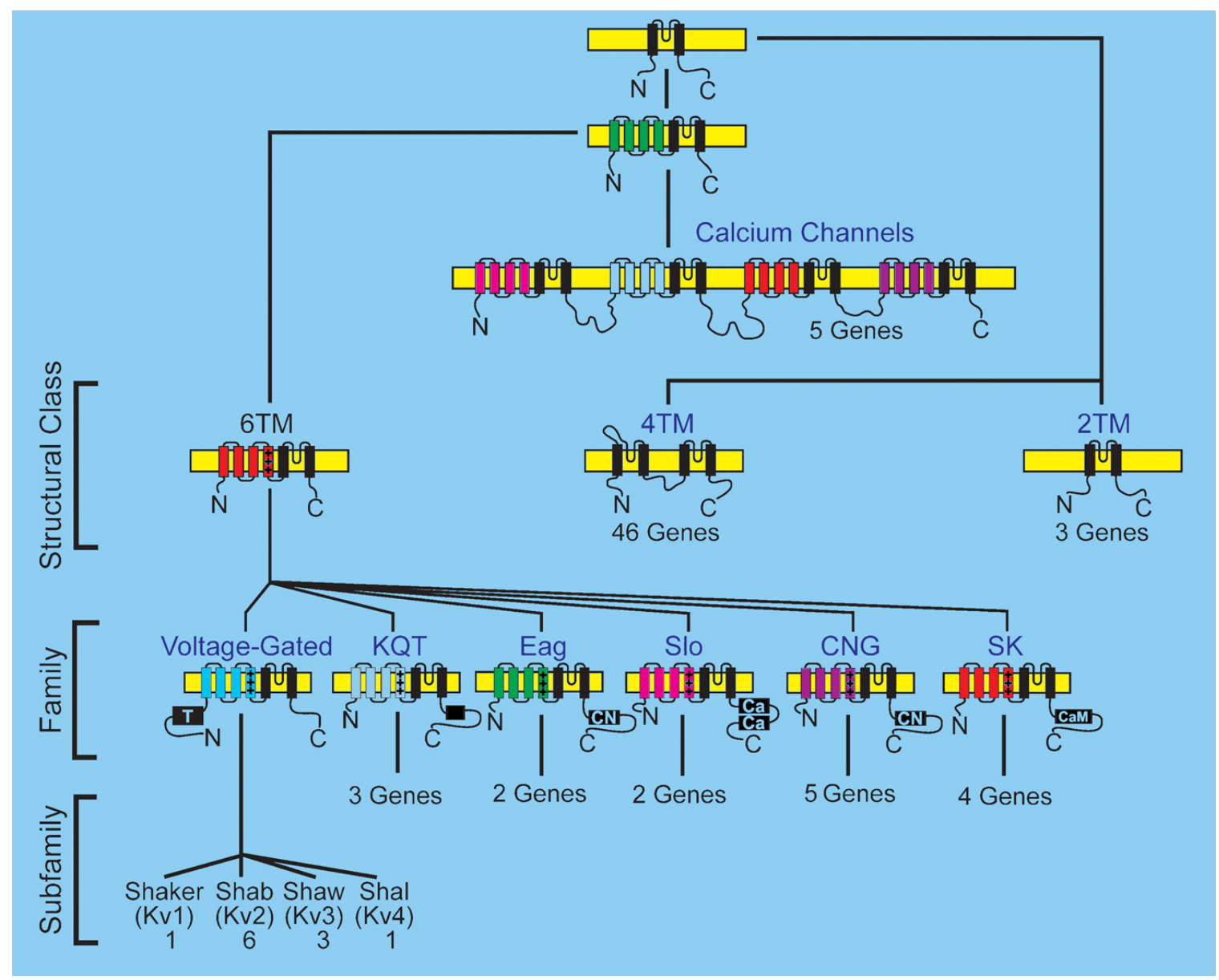

Figure 1. Diagram of potassium channel families and subfamilies. Three structural classes are shown defined by their subunit structure, having six (6TM), four (4TM), or two (2TM) transmembrane segments. The structural class of 6TM channels has six conserved gene families. See: http://nt-salkoff.wustl.edu/ for information about mutant strains and cDNAs for expression studies.

\subsection{Genetic screens for abnormal behavioral phenotypes reveal potassium channel mutants}

An advantage of studying potassium channels in C. elegans is the ease with which the animal is subject to genetic manipulation. Because of the critical involvement of potassium channels in determining the excitability of neurons and muscles, it is perhaps not unexpected that genetic screens based on several C. elegans behavioral phenotypes have yielded examples of mutants linked to nearly every major class of potassium channel subunit. Mutant behavioral phenotypes have included the inability to lay eggs (egl, egg-laying abnormal), uncoordinated body movements (unc, uncoordinated), and abnormal defecation motor programs, in particular failure of the expulsion step (exp, expulsion defective; Brenner, 1974; Trent et al., 1983, Thomas, 1990). Surprisingly, nearly all of these potassium channel mutants were first isolated as dominant alleles that encode gain-of-function channel subunits. Loss-of-function mutants of these subunits, in general, largely resemble wild-type animals. These genetic results are consistent with an interpretation whereby a large degree of functional overlap or redundancy may be shared between wild-type potassium channels to determine normal excitability. The loss of an individual potassium channel may thus be compensated and not result in an overt phenotype. In contrast, any gain-of-function mutation which acts to hyper-activate a potassium channel may inappropriately suppress cellular excitability, causing a dramatic behavioral phenotype. Alternatively, specific functional roles for individual potassium channels may exist, but the deficits resulting from the loss of function of single potassium channel genes may be too subtle to be detected with coarse behavioral assays, and ideal laboratory culturing conditions. These genetic studies have provided insights into important regions of potassium channel subunits involved in gating, as well as potential auxiliary subunits required for activity. Among the 6TM potassium channel subunit genes, six examples are known 
of mutants isolated by forward genetic screens (egl-36[Shaw], egl-2, unc-103[Eag], exp-2 [Shab-like], exp-3 [SK] and slo-1[Slo]). Currently, no mutants have been identified of the $2 \mathrm{TM}$ class of potassium channels but, as will be described, several of the 4TM class have been identified.

\section{The 6TM gene families}

\subsection{Voltage-gated potassium channels}

Voltage-gated potassium channels are the largest class of 6TM channels. These channels are activated by depolarization, but function with a wide diversity of activation and inactivation kinetics. In mammals, these channels are widely expressed in brain, muscle and heart where they control complex waveforms of electrical activity. They are also widely expressed in tissues outside the nervous system such as cells of the immune system (Cahalan and Chandy, 1997). Four conserved subfamilies, first cloned from Drosophila, comprise this family of channels: Shaker (Papazian et al., 1987; Kamb et al., 1987; Pongs et al., 1988), Shab, Shaw and Shal (Wei et al., 1990). Many vertebrate orthologues in each subfamily have been isolated (Chandy and Gutman, 1995). A total of eleven C. elegans genes were identified encoding voltage-gated potassium channel subunits that fall within the four subfamilies of Shaker (Kv1), Shab (Kv2), Shaw (Kv3) and Shal (Kv4). This group includes channels with biophysical properties conserved with other species (Bianchi et al., 2003) as well as those with unexpected biophysical properties (Davis et al., 1999; Fleischhauer et al., 2000). Examples of physiological and genetic studies undertaken with members of this family follow.

Shaw Channels (Kv3 family) egl-36 mutants were isolated from screens for egg-laying deficient mutants. These mutants $(n 728, n 2332)$ are also moderately defective in generating the expulsion step of the defecation motor program, and genetically behave as dominant alleles. Intragenic second mutations that result in null alleles, nearly completely revert the mutant phenotypes. egl-36 encodes a voltage-gated potassium channel subunit which is an ortholog of vertebrate Kv3 (Shaw-like, KCNC) subunits (Elkes, et al., 1997, Johnstone et al., 1997). Heterologous expression studies of EGL-36 channels revealed voltage-gated potassium currents with functional properties similar to vertebrate $\mathrm{Kv} 3$ channels. The two dominant mutant alleles encode missense mutations which alter single residues within the amino-terminal T1 subunit tetramerization domain $(n 728, \mathrm{E} 142 \mathrm{~K})$ and the S6 transmembrane segment (n2332, P438S) of the predicted channel protein. Both mutations cause a hyperpolarizing shift in the voltage-dependence for activation, which could confer abnormal inhibition of cellular excitability. Promoter::GFP fusion experiments indicate expression of egl-36 in uterine and vulva muscles, and in enteric motorneurons (AVL, DVB), consistent with the observed behavioral phenotype.

Shab (Kv2)-like: Exp-2 encodes a potassium channel subunit related to the class of voltage-gated potassium channel subunits, but without a clear vertebrate ortholog (Davis et al., 1999). However, sequence comparisons clearly suggest that it is distantly related to the Shab (Kv2) family. Interestingly, homozygous exp-2 gf animals are inviable and die as young larvae, most likely because they are unable to feed. Heterozygous +lexp-2(gf) animals survive, but are severely expulsion defective, egg-laying defective, and exhibit abnormally brief pharyngeal contractions. Exp-2 null animals grossly resemble wild-type and are viable, but these animals exhibit abnormally prolonged pharyngeal contractions. Promoter::GFP fusion experiments indicate prominent expression of exp-2 in pharyngeal muscles, as well as enteric muscles and sensory neurons in the head and tail ganglia. Heterologous expression studies of EXP-2 channels revealed an unusual voltage-gated potassium channel, which gates by briefly conducting repolarizing current upon hyperpolarizations, but only when preceded by a prior depolarization (Davis et al., 1999; Fleischhauer et al., 2000). This unusual combination of gating properties functionally resembles the vertebrate ether-a-go-go related HERG (KCNH2) potassium channel (Trudeau et al., 1995; Curran et al., 1995; Sanguinetti et al., 1995), but because these two channels share low sequence similarity, this is likely to be an example of convergent evolution. Like HERG channels in vertebrate cardiomyocytes, EXP-2 functions to limit the length of plateau potentials generated by pharyngeal muscle cells, by providing a rapid repolarizing conductance at the end of the plateau potential. The exp-2(sa26) gf mutation alters a single residue in S6 (the sixth membrane spanning segment of the alpha subunit) (C480Y). This mutation shifts the voltage-dependence of activation towards negative potentials, creating a mutant channel that is constitutively active at normal resting potentials (Davis et al., 1999). These exp-2 studies highlight the central importance of S6 structures in controlling the gating of potassium channels, even for functionally and structurally diverse types of channel subunits.

\subsection{KQT potassium channels}

KQT potassium channels are related to the human KvLQT (KCNQ) channel family (Wang et al., 1996), recently implicated in one form of long-QT syndrome, a hereditary cardiac syndrome. There are five of these genes 
in humans (KCNQ1-5). Remarkably, four of the five human genes are associated with hereditary diseases. In addition to long-QT syndrome (associated with KCNQ1), KCNQ mutations have been linked with Benign Familial Neonatal Convulsions (KCNQ2 and KCNQ3; Biervert et al., 1998; Charlier et al., 1998; Singh et al., 1998 and 2003), and the Non-syndromic Autosomal Deafness, DFNA2 (KCNQ4; Kubisch et al., 1999).

C. elegans has three genes encoding channels of the KQT family. The C. elegans homologue of the human cardiac KvLQT1 channel (KQT-1) may have a conserved role that offers insight into its function in both species. As discussed previously, the orthologues in humans (called the KCNQ family) are the loci of many genetic diseases. Human KCNQ channels are expressed in both cardiac cells where they limit the length of the plateau phase of the cardiac action potential, and in the intestine where they may help provide the negative membrane potential necessary for apical chloride efflux mediating fluid secretion. In $C$. elegans genes of the KQT family are expressed both in the pharynx which has many cardiac-like electrical properties (Avery and Horvitz, 1989; Raizen and Avery, 1994) and also in the intestine (figure 2). Deletions of these genes have now been isolated (Wei et al., 2002) which may help in the further analysis of their physiological roles in C. elegans.

A

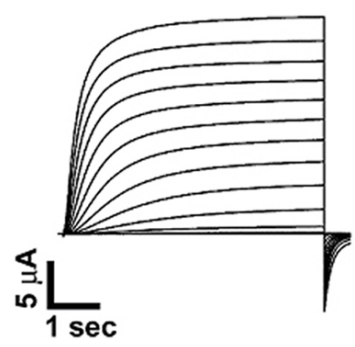

KQT-1

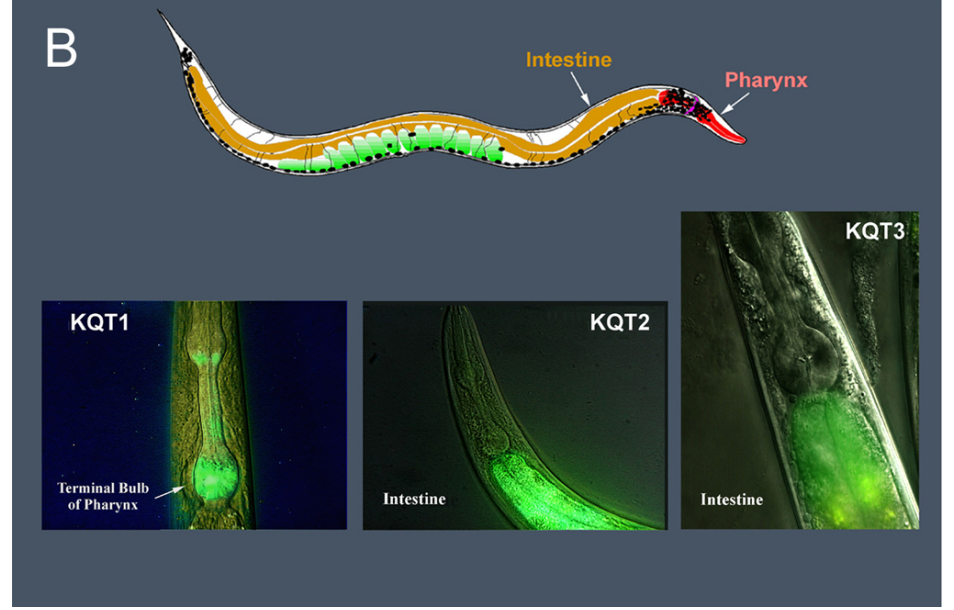

KQT-3

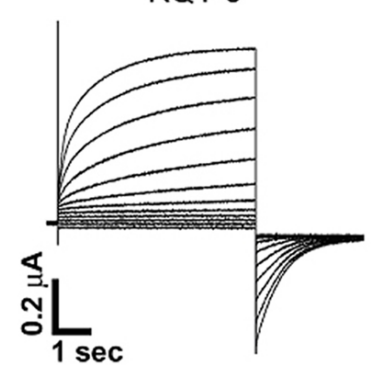

action potentials in the pharynx

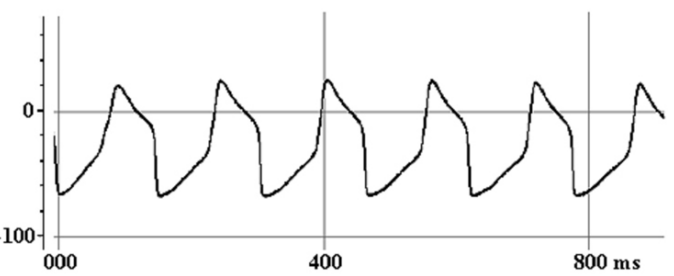

Figure 2. A. Functional expression of C. elegans KQT cDNA's in Xenopus oocytes using the two electrode voltage-clamp technique; test pulses were applied at $10 \mathrm{mV}$ intervals from a holding potential of $-80 \mathrm{mV}$. The slow onset of the voltage-dependent current resembles that of human KCNQ counterparts (Wei et al., 2005). B. Tissue specific expression patterns in the cardiac-like pharynx and intestine as revealed by Promoter-GFP expression experiments. C. Cardiac-like action potentials in the C. elegans pharynx (Salkoff, unpublished; see also Raizen and Avery, 1994 and Shtonda and Avery, 2005).

\subsection{Eag-like potassium channels}

First discovered in Drosophila (Warmke et al., 1991), these channels form a diverse family (Warmke and Ganetzky, 1994) that may be divided into three distinct subfamilies (Eag, Erg and Elk). One human orthologue, Herg, with unusual gating properties, is also implicated in long-QT syndrome (Smith et al., 1996; Trudeau et al., 1995; Curran et al., 1995). C. elegans has two genes of this family, one which is an eag orthologue (egl-2) and one which is an erg orthologue (unc-103). Similar genetic and functional studies have been observed for mutants of both (Wienshenker et al., 1999; Reiner et al., 1999; Petersen et al., 2004). Egl-2 gf mutants exhibit pleiotropic behavioral defects. In addition to egg-laying deficiency, these mutants show sluggish movement, defective expulsion- 
defecation cycles, and more subtle defects in mechanosensation and chemosensation. Two independently isolated $g f$ alleles of egl-2 (n693, n2656) encode the same missense mutation, altering a single highly conserved residue (A533V) in the S6 transmembrane segment of the channel subunit. Heterologous expression studies confirmed that this mutation shifts the voltage-dependence of activation for this channel towards hyperpolarized potentials, allowing these mutant channels to abnormally inhibit excitability. Promoter::GFP fusion experiments indicate expression in vulva muscles, enteric muscles, mechanosensory and chemosensory neurons. Likewise unc-103(e1597) gf mutants exhibit behavioral defects resulting from a missense mutation altering a similar conserved residue in S6 (A334T), though functional analysis of the UNC-103 channel by electrophysiological studies has not been reported. Though unc-103 null hermaphrodites are grossly wild-type, male worms carrying homozygous null mutations of unc-103 show abnormal spicule protraction, consistent with hyperactive protractor muscle activity (Garcia and Sternberg, 2003). In addition, a genetic interaction between UNC-103 channels and calcium/calmodulin-dependent kinase II (CaMKII) (encoded by unc-43) is suggested, because the unc-103 null mutation suppresses expulsion defects caused by an unc-43(n498) $g$ f mutation (Reiner et al., 1999).

\subsection{Calcium-activated Slo-like potassium channels}

Channels of this family, were also first cloned in Drosophila (Elkins et al., 1986: Atkinson et al., 1991) and form large conductance 'BK-type' channels, gated by both voltage and intracellular calcium (Adelman et al., 1992; Butler et al., 1993). This class can be divided into three subfamilies, typified by the vertebrate genes $m S l o$ (Slo- 1 ), Slack (Slo-2) and mSlo-3 (Slo-3). In mammals, Slo-1 and Slack (Slo-2) are expressed in brain, various muscles, and most cell types that also express voltage-dependent calcium channels. Slo- 1 channels control the myogenic tone of arterial and airway smooth muscle, and are also key regulators of neurotransmitter release. C. elegans possess a single Slo-1 orthologue (slo- 1 ) that plays an important regulatory role in synaptic transmission (Wang et al., 2001). Slo-2 is a somewhat more distantly related C. elegans Slo member; its vertebrate orthologue is the Slack gene (Joiner et al., 1998, Yuan et al., 2003). Interestingly, the slo-2 gene expresses large conductance potassium channels with sensitivity to both intracellular calcium and chloride ions (Yuan et al., 2000).

In contrast to other potassium channel mutants, slo- 1 mutants were isolated as recessive loss-of-function mutations, from two independent forward genetic screens. Phenotypically, slo-1 lof mutants are grossly wild-type, though their movement is slightly jerky, and they exhibit decreased resistance to aldicarb paralysis compared to wild-type animals, consistent with increased cholinergic synaptic transmission.

Mutants of SLO-1 potassium channels were useful tools to show that the SLO-1 channel regulates the quantal content of neurotransmitter release. The absence of electrophysiological techniques to characterize synaptic physiology had been a handicap in studying synaptic function in $C$. elegans until the innovative studies by Janet Richmond (Richmond and Jorgensen, 1999). The techniques developed for those studies were further developed by Wang et al. (2001) to permit quantal analysis of neurotransmitter release. Large conductance $\mathrm{Ca}^{2+}$-activated $\mathrm{K}^{+}$ channels (Slo-1) have been implicated in the control of neurotransmitter release in vertebrates by pharmacological methods. Mutant analysis and physiological methods in C. elegans similarly showed that slo- 1 controls the quantal content of neurotransmitter release. Six Slo-1 mutants were isolated in a screen for suppressors of a hypomorphic syntaxin mutant (unc-64), a mutation that confers behavioral lethargy. The selection of suppressor mutations was based on the reversal of lethargy. The quantal content of evoked excitatory postsynaptic currents (EPSCs) in unc-64 mutants was $\sim 5 \%$ of wild-type, but the addition of a slo- 1 mutation increased this fourfold. Slo- 1 mutations were also found to increase the quantal content of release even in a wild-type (unc-64+) background (Wang et al., 2001). Independently, multiple slo-1 lof mutants were also isolated from a screen for resistance to acute ethanol intoxication (Davies et al., 2003). This study argued that SLO-1 channels are specific targets for up-regulation by ethanol, which could mediate the suppression of movement caused by ethanol intoxication.

Slo-2, a BK channel requiring both chloride and calcium (Yuan et al., 2000; Jospin et al., 2002). Potassium channels have been reported to be modulated by many diverse factors, but modulation of gating by intracellular chloride ion has previously been unsuspected. However, when slo-2, was cloned in C. elegans and expressed in the heterologous Xenopus oocyte expression system, it was found to have a novel requirement for both chloride and calcium; no activity is detectable in the absence of either. The sequence divergence between slo- 2 and slo- 1 may underlie some of their functional differences, especially in the "tail" domain of the channel where calcium-sensing may occur. The unique property of chloride dependence has never been described for any other ion channel. Perhaps the SLO-2 channel is an intracellular $\mathrm{Cl}^{-}$sensor linking changes in $\mathrm{Cl}_{\mathrm{i}}^{-}$with membrane electrical behavior. $C$. elegans transformed with a slo-2 promotor::GFP transgene revealed that slo-2 is widely expressed in both neurons and muscles and is highly abundant as suggested by the large number of ESTs. In muscle cells the SLO-2 current has been shown to be larger than the voltage-dependent potassium currents which are contributed by Shaker (Kv1) 
and Shal (Kv4) channel types (Santi et al., 2003). Intriguingly, the slo-2 tissue expression pattern overlaps with that of slo- 1 suggesting that the two subunits might form functional heteromeric channels in vivo. The rat homologue of SLO-2 has been reported to possibly form heteromultimers with the mammalian BK channel, mSLO-1 (Joiner et al., 1998). SLO-2's dependence on $\mathrm{Cl}^{-}$suggests that intracellular $\mathrm{Cl}^{-}$homeostasis may play an important role in regulating cellular excitability through this unusual $\mathrm{K}^{+}$channel. Recently, the mammalian orthologue of the SLO-2 channel was shown to be a sodium-activated potassium channel (which is also co-activated by chloride ions) (Yuan et al., 2003). The sodium-activated potassium channel was originally identified in cardiac myocytes and thought to confer resistance to hypoxia. Interestingly, slo-2 mutants in $C$. elegans are especially susceptible to hypoxia, suggesting a conserved role for Slo-2 channels. Another point of interest is the fact that calcium and chloride ions co-activate SLO-2 channels in C. elegans, while sodium and chloride ions co-activate SLO-2 channels in mammals. This may be an evolutionary adaption reflecting the absence of the classical voltage-dependent sodium channel in $C$. elegans.

\subsection{Cyclic nucleotide-gated cation channels}

These channels are gated by intracellular cyclic nucleotides (Kaupp et al., 1989), and are expressed in receptor neurons of vertebrate retina and olfactory epithelium, where they transduce primary sensory information into changes in membrane potential. This transduction process is mediated by the intracellular messengers cAMP (olfactory receptors; Goulding et al., 1992), or cGMP (visual photoreceptors; Kaupp et al., 1989). Though these channels are non-selective cation channels (Heginbotham et al., 1992), they are structurally similar to 6TM potassium channels and are considered here as a related family. Five $C$. elegans genes encode 6TM subunits with high sequence conservation to cyclic nucleotide-gated cation channels in other species. C. elegans with mutations in either of two cyclic nucleotide-gated channel genes (tax-2 and tax-4) result in animals which are defective in chemotaxis and thermotaxis (Coburn and Bargmann 1996; Komatsu et al., 1996).

\subsection{SK Small conductance, voltage insensitive calcium-activated potassium channels}

Small-conductance calcium-activated potassium channels (SK channels) are fundamental regulators of excitability. Unlike high conductance calcium-activated potassium channels which are activated by both voltage and calcium ions, SK channels are activated solely by intracellular calcium ions and therefore may function at rest or at hyperpolarized potentials. SK channels underlie the slow after-hyperpolarization (sAHP) following an action potential. Regulation of the sAHP represents a powerful modulatory influence on excitability. In addition to calcium, many neurotransmitters act on the sAHP. When these channels are present, changes in intracellular calcium exert strong effects on neuronal excitability. SK channels are also found in cells of the immune system. There are four genes encoding SK channels in C. elegans, one of which appears to be the locus of the exp-3 mutation. exp-3 mutants exhibit a similar phenotype to other described dominant potassium channel mutants. Mutant exp-3(n2372) $g f$ animals are severely defecation-expulsion and egg-laying defective (Thomas, 1990). We have recently mapped exp-3 to B0399.1, which is the $C$. elegans ortholog of a vertebrate SK gene that encodes a small-conductance calcium activated potassium channel subunit (Salkoff et al., unpublished). We have generated a null deletion of this gene. Similar to other potassium channel mutants, homozygous null animals grossly resemble wild-type, though there may be a slight temperature-dependent larval lethality at higher temperatures $\left(>20^{\circ} \mathrm{C}\right)$.

\section{2TM potassium channels}

Three $C$. elegans genes, $n I R K 1-3$ have the same subunit structure as inward rectifier potassium channel subunits from other species. Vertebrate 2TM inward rectifier subunits can be grouped into at least six subfamilies termed Kir1 through Kir6 (Doupnik et al., 1995). However, the two nematode nIRK genes appear more closely related to each other than to any of the vertebrate genes. This raises the question of whether vertebrate $2 \mathrm{TM}$ subfamilies have specific homologues in C. elegans and other invertebrates, or if they differentiated in the vertebrate line of evolution. No mutants have yet been reported to be associated with any of the three genes encoding $2 \mathrm{TM}$ potassium channel subunits.

\section{4TM potassium channels}

Surprisingly, the most frequently encountered type of potassium channel belongs to the 4TM class of channel. Forty six genes of this class are currently known. It has been suggested that this large number may reflect a special adaptation of $C$. elegans to customize the electrical properties of individual cell types (Salkoff et al., 2001). In Drosophila there are approximately eleven genes encoding channels of this type (Adams et al., 2000; Littleton and Ganetzky, 2000; and Rubin et al., 2000), while in humans there are approximately fifteen genes. 
Four mutants are known of the 4TM "TWK" subunit class (sup-9, twk-18, unc-58, egl-23). sup-9 encodes TWK-38 (de la Cruz et al., 2003), which is a clear structural orthologue of the human TASK (KCNK9) gene (Duprat, et al., 1997). SUP-9 likely functions in a native channel complex with at least two other gene products (SUP-10 and UNC-93), based on extensive genetic evidence (de la Cruz, et al., 2003; Levin and Horvitz, 1993; Levin and Horvitz, 1992). unc-93 is a member of an extended gene family encoding multipass membrane proteins conserved among C. elegans, Drosophila, and humans. This family includes the sulfonylurea receptor that forms a channel complex with the vertebrate $\mathrm{K}_{\mathrm{ATP}}$ channel (see below). SUP-10 is a unique putative membrane protein. Gain-of-function alleles of sup-9, unc-93 or sup-10 all cause a similar behavioral phenotype, characterized by sluggish body movements, egg-laying and defecation defects, and a "rubberband" uncoordinated response to prodding of the head, whereas loss-of-function mutants resemble wild-type. However, loss of function of any gene in this group suppresses the mutant phenotype caused by a gain of function of either of the remaining two genes. Genetic suppression of sup- $9(g f)$ is observed by the loss of unc-93 function, even when sup- $9(g f)$ is overexpressed as an extrachromosomal array, suggesting an absolute requirement for both gene products (de la Cruz, et al., 2003). Taken together, these results argue that SUP-9, UNC-93, and SUP-10 combine to form a native channel complex, perhaps analogous to the vertebrate $\mathrm{K}_{\mathrm{ATP}}$ channel complex formed by 2TM Kir6.2 potassium channel subunits and the SUR sulfonylurea receptor (Auilar-Bryan, et al., 1995; Inagaki, et al., 1996; Shyng and Nichols, 1997). The sup-9(n1550) gain-of-function allele encodes a missense mutation that substitutes threonine for alanine 236 in the fourth transmembrane segment of the subunit. This presumably acts to stabilize the open conformation of the channel. Electrophysiological analysis of the putative SUP-9 channel complex, however, has not been reported thus far by heterologous expression studies, and may require in situ recordings from C. elegans cells.

By contrast, combined genetic and electrophysiological analyses of $t w k-18$ mutants have been possible. Two separate dominant alleles of $t w k-18$ (also referred to as unc-110 and mah-2 in the literature; Reiner, et al., 1995 and Hosono, et al., 1985, respectively) were isolated from independent behavioral screens for uncoordinated movement (e1913) and temperature-sensitive paralysis (cn110). Homozygous $t w k-18(e 1913)$ animals die as embryonic lethals, but heterozygous $t w k-18(e 1913) /+$ animals display flaccid paralysis and uncoordinated movement. The second allele, cn110, is viable as a homozygous animal and moves well at lower temperatures $\left(15^{\circ}-22^{\circ} \mathrm{C}\right)$, but becomes uncoordinated and paralyzed at elevated temperatures $\left(>22^{\circ} \mathrm{C}\right) . T w k-18$ encodes a $4 \mathrm{TM}$ potassium channel subunit without a clear vertebrate ortholog (Kunkel, et al., 2000), similar to most of the $\sim 46$ C. elegans twk genes. Expression of $t w k-18$ is confined to bodywall muscles based on promoter::GFP reporter studies, consistent with its mutant behavioral phenotype. Electrophysiological analysis of TWK-18 by heterologous expression studies in Xenopus oocytes revealed non-inactivating potassium-selective currents with little voltage-dependence, but activated by elevated temperatures $\left(>23^{\circ} \mathrm{C}\right)$, with a steep temperature-dependence. The effect of the gain-of-function mutations is a 4- to 5-fold increase in the level of currents measured in Xenopus oocytes without an obvious change in unitary conductance through single channels. However, the exact mechanism of this increase remains unresolved. The gain-of-function alleles encode missense mutations G165D (e1913) at the base of the second transmembrane segment, and M280I (cn110) at the top of the fourth transmembrane segment of the subunit, suggesting that these regions of the channel subunit may be critical for gating.

Further genetic analyses of two behavioral mutants have identified linkages to two additional 4TM TWK subunits, unc-58 (encoding TWK-19) and egl-23 (encoding TWK-41). As with sup-9 and twk-18, these additional mutants were isolated as semidominant alleles which were reverted by intragenic second mutations that render the genes effectively null. These null mutants grossly resemble wild-type animals. The gain-of-function unc-58(e665) mutant displays rigid paralysis with a characteristic "shaking" phenotype, constitutive egg-laying, and short "dumpy" body length consistent with hypercontracted bodywall muscles. This mutant phenotype is suppressible by treatment with the pesticide endosulfan which may directly block the UNC-58 channel (Tzoneva and Thomas, personal communications). Promoter::GFP fusion studies indicate expression of unc-58 in ventral cord motorneurons and interneurons (Salkoff et al., 2000). These results are consistent with an unc-58 gf mutation which alters ionic selectivity, perhaps analogous to the weaver mutation of GIRK2 (Silverman, et al., 1996), which converts an inhibitory potassium-selective channel to an excitatory non-selective cation channel. Egl-23(n601) $g f$ mutant animals are defective in locomotion, egg-laying and enteric muscle contractions. The egl-23(n601) mutant phenotype can be reverted by intragenic second mutations, or by RNAi (Johnstone and Thomas, personal communications). Electrophysiological analyses of the channels encoded by either unc-58 or egl-23 have not yet been reported. 


\subsection{Evolution may tune the excitability of individual neurons using 4TM channels}

The relationship between the genome and the evolution of the nervous system may differ between an animal like $C$. elegans with 302 neurons, and mammals with tens of billions of neurons. It was found that the 4TM class of nonconserved potassium channels is highly expanded in C. elegans and may play a special role in the evolution of its nervous system. The $C$. elegans genome contains an extended gene family of potassium channels whose members fall into two evolutionary divergent classes. One class constitutes an ancient conserved "set" of K+ channels with orthologues in both humans and Drosophila; a second larger class is made up of rapidly evolving genes unique to $C$. elegans. Chief among this second class are the novel "TWK" (4TM) type potassium channels that may function as regulated leak conductances to modulate cell electrical excitability. This inventory of TWK channels is far larger in $C$. elegans than in humans or Drosophila. Evidence for their rapid evolution is the observation that they tend to be less conserved between $C$. elegans and a sibling species, $C$. briggsae, than are voltage-dependent channels, like Shaker. Unlike conserved channel genes, many TWK genes are expressed in very few cells. DNA enhancer elements associated with these genes were also identified that direct gene expression to individual neurons. C. elegans may maintain an exceptionally large inventory of novel TWK channels (as well as ligand-gated channels) as an adaptive mechanism to "fine tune" the electrical properties of individual neurons, making the most of its limited circuitry (Salkoff et al., 2001).

\section{Usefulness of the information in C. elegans}

\subsection{C. elegans can be a useful system to dissect apart the components of cell excitability using its versatile genetic and molecular resources}

GFP-promoter experiments showed that at least nine genes encoding potassium channel subunits are expressed in C. elegans muscle. By using genetic, RNAi and physiological techniques, the molecular identity of all major components of the outward $\mathrm{K}^{+}$currents in body wall muscle cells in culture and in situ, were revealed (Jospin et al. 2002; Santi et al. 2003). Under physiological conditions, outward current is dominated by the products of only two genes, Shaker (Kv1) and Shal (Kv4), both expressing voltage-dependent potassium channels. Both of these voltage-dependent currents can be removed either by the use of mutants or by treating cultured cells with appropriate RNAi's. GFP-promoter experiments also indicated that the high conductance $\mathrm{Ca}^{2+} \& \mathrm{Cl}^{-}$activated SLO-2 channels are prominently expressed. A deletion mutant was generated to identify the SLO-2 current in native cells. It was shown that native SLO-2 channels were active only when intracellular $\mathrm{Ca}^{2+}$ and $\mathrm{Cl}^{-}$were raised above normal physiological conditions, as occurs during hypoxia. However, under such conditions, SLO-2 is the largest outward current, contributing up to $87 \%$ of the total current. Other genes encoding potassium channels are expressed in muscle, but do not appear to contribute a large component of total outward current under physiological conditions. However they may contribute currents conditional on other factors such as temperature, or stringent conditions of salinity, osmolarity or $\mathrm{pH}$. Hence, excitable membranes may contain a complex combination of household conductances functioning under normal circumstances, with additional conductances that are activated during unusual physiological circumstances.

\subsection{Conserved genes represent natural structural variants}

The large number of conserved genes encoding ion channels in C. elegans represent structural variants useful to: 1. Biophysicists, protein structure analysts and X-ray crystallographers. Similarities and differences in biophysical properties between species can be mapped in structure-function studies to gain insight into mechanisms and structural domains responsible for particular properties. 2. Pharmacologists. In an analogous manner similarities and differences in pharmacological properties between the $C$. elegans and vertebrate channels can be mapped in structure-function studies to gain insight into binding sites and mechanisms of channel-drug interactions.

\subsection{C. elegans could be a useful model system for studying genetic disease which involves ion channels}

Hereditary diseases involving ion channels cover various fields of medicine such as neurology (nocturnal frontal lobe epilepsy, benign neonatal convulsions, episodic ataxia, hemiplegic migraine, deafness, stationary night blindness), nephrology (X-linked recessive nephrolithiasis, Bartter), myology (hypokalemic and hyperkalemic periodic paralysis, myotonia congenita, paramyotonia, malignant hyperthermia), and cardiology (LQT syndrome). All types of voltage-gated ion channels for cations (sodium, calcium, and potassium channels) and anions (chloride channels) are involved (reviewed in Lehmann-Horn and Jurkat-Rott, 1999). It is difficult to predict all the ways in which $C$. elegans might be useful in contributing to a better understanding of hereditary disease. For example, the functions of the polycystins and the molecular basis of kidney cystogenesis are now better understood thanks to 
research done in C. elegans (Barr and Sternberg, 1999). One polycystin was discovered to be an ion channel. Based on the role of polycystins in $C$. elegans sensory neuron function and the conservation of PKD pathways it is now proposed that polycystins act as sensors of the extracellular environment, mediating intracellular transduction events which are important in both physiological and morphogenetic processes (Kaletta et al., 2003).

\subsection{The study of ion channel functional properties in $C$. elegans has revealed unusual properties never before seen}

Potassium channels have many varied properties, some of which were seen for the first time in C. elegans. Two examples are a temperature-sensitive potassium channel (Kunkel et al., 2000, and a chloride-sensitive potassium channel (Yuan et al., 2000). These findings may encourage further studies in the perception and sensation of temperature-induced pain, or studies of how cells and tissues compensate for temperature-changes. The discovery of a chloride sensitive channel may encourage further studies of the role of chloride in controlling membrane potential and in repolarizing active responses. Since the mammalian orthologue of Slo-2 is expressed in the kidney, its role in kidney function vis-à-vis its possible chloride sensitivity may also be an important area of investigation.

\subsection{Mutant analysis in $C$. elegans has revealed gain-of-function mutants with possible therapeutic uses}

In studying several instances of mutant potassium channels in $C$. elegans, several examples of gain-of-function mutants were uncovered that suggest ways in which transgenic techniques may be useful in future clinical applications. These are mutants in which potassium channels are open inappropriately either because their voltage range of activation has been shifted negatively, or because their activity level is abnormally high for other reasons. These results could guide the construction of vectors designed to electrically silence cells, possibly without detrimental effects on cell viability. The introduction of such vectors into selected sets of sensory neurons in mammals, such as C-fibers, may offer an effective remedy for such conditions as chronic pain. Naturally such uses have to wait for the development of tissue-specific promoters and viral or "gene-gun" techniques for the introduction of vectors into cell populations, but $C$. elegans may also offer an effective system for "proof-of-principle" experiments.

\section{Acknowlegments}

Supported by grants R24 RR017342-01 and R01 GM067154-01A1 from the National Institutes of Health to LS, and IBN-0117341 from the National Science Foundation to AW. Special thanks to Michael Nonet for many helpful suggestions.

\section{References}

Adams, M.D., et al. (2000). The genome sequence of Drosophila melanogaster. Science 287, 2185-2195. Abstract Article

Adelman, J.P., Shen, K.Z., Kavanaugh, M.P., Warren, R.A., Wu, Y.N., Lagrutta, A., Bond, C.T., and North, R.A. (1992). Calcium-activated potassium channels expressed from cloned complementary DNAs. Neuron 9, $209-216$. Abstract Article

Aguilar-Bryan, L., Nichols, C.G., Wechsler, S.W., Clement, J.P.T., Boyd, A.E. III, Gonzalez, G., Herrera-Sosa, H., Nguy, K., Bryan, J., and Nelson, D.A. (1995). Cloning of the beta cell high-affinity sulfonylurea receptor: a regulator of insulin secretion. Science 268, 423-426. Abstract

Anderson, J.A., Huprikar, S.S., Kochian, L.V., Lucas, W.J., and Gaber, R.F. (1992). Functional expression of a probable Arabidopis thaliana potassium channel in Saccharomyces cerevisiae. PNAS 89, 3736-3740. Abstract

Atkinson, N.S., Robertson, G.A., and Ganetzky, B. (1991). A component of calcium-activated potassium channels encoded by the Drosophila Slo locus. Science 253, 551-555. Abstract

Bargmann, C.I. (1998). Neurobiology of the Caenorhabditis elegans genome. Science 282, 2028-2033. Abstract Article 
Barr, M.M., and Sternberg, P.W. (1999). A polycystic kidney-disease gene homologue required for male mating behavior in C. elegans. Nature 401, 386-389. Abstract Article

Bianchi, L., Kwok, S.M., Driscoll, M., and Sesti, F. (2003). A potassium channel-MiRP complex controls neurosensory function in Caenorhabditis elegans. J. Biol. Chem. 278, 12415-12424. Abstract Article

Biervert, C., Schroeder, B.C., Kubisch, C., Berkovic, S.F., Propping, P., Jentsch, T.J., and Steinlein, O.K. (1998). A potassium channel mutation in neonatal human epilepsy. Science 279, 403-406. Abstract Article

Brenner, S. (1974). The genetics of Caenorhabditis elegans. Genetics 77, 71-94. Abstract

Butler, A., Tsunoda, S., McCobb, D.P., Wei, A., and Salkoff, L. (1993). mSlo, a complex mouse gene encoding "maxi" calcium-activated potassium channels. Science 261, 221-224. Abstract

Cahalan, M.D., and Chandy, K.G. (1997). Ion channels in the immune system as targets for immunosuppression. Curr. Opin. Biotechnol. 8, 749-756. Abstract

C. elegans Sequencing Consortium. (1998). Genome sequence of the nematode C. elegans: A platform for investigating biology. Science 282, 2012-2018. Article

Chandy, K.G., and Gutman, G.A. (1995). Voltage-gated K+ channel genes. In: CRC Handbook of Receptors and Channels, P.A. North, ed., CRC Press, Boca Raton, FL, pp. 1-71.

Charlier, C., Singh, N.A., Ryan, S.G., Lewis, T.B., Reus, B.E., Leach, R.J., and Leppert, M. (1998). A pore mutation in a novel KQT-like potassium channel gene in an idiopathic epilepsy family. Nat. Genet. 18, 53-55. Abstract Article

Coburn, C.M., and Bargmann, C.I. (1996). A putative cyclic nucleotide-gated channel is required for sensory development and function in C. elegans. Neuron 17, 695-706. Abstract Article

Connor, J.A., and Stevens, C.F. (1971). Voltage-clamp studies of a transient outward membrane current in gastropod neural somata. J. Physiol. 213, 21-30. Abstract

Connor, J.A. (1980). The fast $\mathrm{K}+$ Channel and Repetitive Firing. In: Cold Spring Harbor Reports in the Neurosciences, Vol. 1, J. Koester and J. Byrne, eds., CSHL, pp. 125-133.

Curran, M.E., Splawski, I., Timothy, K.W., Vincent, G.M., Green, E.D., and Keating, M.T. (1995). A molecular basis for cardiac arrhythmia: HERG mutations cause long QT syndrome. Cell 80, 795-803. Abstract Article

Davies, A.G., Pierce-Shimomura, J.T., Kim, H., VaHoven, M.K., Thiele, T.R., Bonci, A., Bargmann, C.I., and McIntire, S.L. (2003). A central role of the BK potassium channel in behavioral responses to ethanol in C. elegans. Cell 115, 655-666. Abstract Article

Davis, M.W., Fleischhauer, R., Dent, J.A., Joho, R.H., and Avery, L. (1999). A mutation in the C. elegans EXP-2 potassium channel that alters feeding behavior. Science 286, 2501-2504. Abstract Article

De la Cruz, I.P., Levin, J.Z., Cummins, C., Anderson, P., and Horvitz, H.R. (2003). Sup-9, sup-10 and unc-93 may encode components of a two-pore $\mathrm{K}+$ channel that coordinates muscle contraction in Caenorhabditis elegans. J. Neurosci. 23, 9133-9145. Abstract

Doyle, D.A., Morais Cabral, J., Pfuetzner, R.A., Kuo, A., Gulbis, J.M., Cohen, S.L., Chait, B.T., and MacKinnon, R. (1998). The structure of the potassium channel: molecular basis of K+ conduction and selectivity. Science 280, 69-77. Abstract Article

Doupnik, C.A., Davidson, N., and Lester, H.A. (1995). The inward rectifier potassium channel family. Curr. Opin. Neurobiol. 5, 268-277. Abstract Article

Duprat, F., Lesage, F., Fink, M., Ryeyes, R., Heurteaux, C., and Lazdunski, M. (1997). TASK, a human background $\mathrm{K}+$ channel to sense external $\mathrm{pH}$ variations near physiological pH. EMBO J. 16, 5464-5471. Abstract Article 
Elkes, D.A., Cardozo, D.L., Madison, J., and Kaplan, J.M. (1997). EGL-36 Shaw channels regulate C. elegans egg-laying muscle activity. Neuron 19, 165-174. Abstract Article

Elkins, T., Ganetzky, B., and Wu, C.F. (1986). A Drosophila mutation that eliminates a calcium-dependent potassium current. PNAS 83, 8415-8419. Abstract

Fleischhauer, R., Davis, W., Dzhura, I., Neely, A., Avery, L., and Joho, R. (2000). Ultra-fast inactivation causes inward rectification in a voltage-gated $\mathrm{K}+$ channel from C.elegans. J. Neurosci.

Garcia, L.R., and Sternberg, P.W. (2003). Caenorhabditis elegans UNC-103 ERG-like potassium channel regulates contractile behaviors of sex muscles in males before and during mating. J. Neurosci. 23, 2696-2705. Abstract

Goulding, E.H., Nagi, J., Kramer, R.H., Colicos, S., Axel, R., Siegelbaum, S.A., and Chess, A. (1992). Molecular cloning and single-channel properties of the cyclic nucleotide-gated channel from catfish olfactory neurons. Neuron 8, 45-58. Abstract Article

Heginbotham, L., Abramson, T., and MacKinnon, R. (1992). A functional connection between the pores of distantly related ion channels as revealed by mutant $\mathrm{K}+$ channels. Science 258, 1152-1155. Abstract

Heginbotham, L., Lu, Z., Abramson, T., and MacKinnon, R. (1994). Mutations in the K+ channel signature sequence. Biophys. J. 66, 1061-1067. Abstract

Hosono, R., Juno, S., and Midsukami, M. (1985). Temperature-sensitive mutations causing reversible paralysis in Caenorhabditis elegans. J. Exp. Zool. 235, 409-421. Abstract Article

Inagaki, N., Gonoi, T., Clement, J.P., Wang, C.Z., Aguilar-Bryan, L., Bryan, J., and Seino, S. (1996). A family of sulfonylurea receptors determines the pharmacological properties of ATP-sensitive K+ channels. Neuron 16, 1011-1017. Abstract Article

Jegla, T., and Salkoff, L. (1995). A multigene family of novel K+ channels from Paramecium tetraurelia. Receptors Channels 3, 51-60. Abstract

Jiang, Y., Lee, A., Chen, J., Cadene, M., Chait, B.T., MacKinnon, R. (2002). Crystal structure and mechanism of a calcium-gated potassium channel. Nature 417, 515-522. Abstract Article

Johnstone, D., Wei, A., Butler, A., Salkoff, L., and Thomas, J. (1997). Behavioral defects in C. elegans egl-36 mutants result from potassium channels shifted in voltage-dependence of activation. Neuron 19, 151-164. Abstract Article

Joiner, W.J., Tang, M.D., Wang, L.Y., Dworetzky, S.I., Boissard, C.G., Gan, L., Gribkoff, V.K., and Kaczmarek, L.K. (1998). Formation of intermediate-conductance calcium-activated potassium channels by interaction of SLACK and SLO subunits. Nature Neurosci. 1, 462-469. Abstract Article

Jospin, M., Mariol, M.C., Segalat, L., and Allard, B. (2002). Characterization of K(+) currents using an in situ patch clamp technique in body wall muscle cells from Caenorhabditis elegans 1. J. Physiol. 544(Pt 2), 373-384. Abstract Article

Kaletta, T., Van der Craen, M., Van Gell, A., Dewulf, N., Bogaret, T., Branden, M., King, K.V., Buechner, M., Barstead, R., Hyink, D., Li, H.P., Geng, L., Burrow, C., and Wilson, P. (2003). Towards understanding the polycystins. Nephron. Exp. Nephrol. 93, e9-e17. Abstract Article

Kamb, A., Iverson, L.E., and Tanouye, M.A. (1987). Molecular characterization of Shaker, a Drosophila gene that encodes a potassium channel. Cell 50, 405-413. Abstract Article

Kaupp, B.U., Niidome, T., Tanabe, T., Terada, S., Bonigk, W., Stühmer, W., Cook, N., Kangawa, K., Matsuo, H., Hirose, T., and Numa, S. (1989). Primary structure and functional expression from complementary DNA of the rod photoreceptor cyclic GMP-gated channel. Nature 342, 762-766. Abstract Article

Katz, B. (1966). Nerve, Muscle, and Synapse. McGraw-Hill, New York. 
Ketchum, K.A., Joiner, W.J., Sellers, A.J., Kaczmarek, L.K., and Goldstein, S.A.N. (1995). A new family of outwardly rectifying potassium channel proteins with two pore domains in tandem. Nature 376, 690-695. Abstract Article

Ketchum, K.A., and Slayman, C.W. (1996). Isolation of an ion channel gene from Arabidopis thaliana using the H5 signture sequence from voltage-dependent $\mathrm{K}^{+}$channels. FEBS Lett. 378, 19-26. Abstract Article

Koh, S.D., Campbell, J.D., Carl, A., and Sanders, K.M. (1995). Nitric oxide activates multiple potassium channels in canine colonic smooth muscle. J. Physiol. 489, 735-43. Abstract

Kohler, M., Hirschberg, B., Bond, C.T., Kinzie, J.M., Marrion, N.V., Maylie, J., and Adelman, J.P. (1996). Small-conductance, calcium-activated potassium channels from mammalian brain. Science 273, 1709-1714. Abstract

Komatsu, H., Mori, I., Rhee, J.S., Akaike, N., and Ohshima, Y. (1996). Mutations in a cyclic nucleotide-gated channel lead to abnormal thermosensation and chemosensation in C. elegans. Neuron 17, 707-718. Abstract Article

Kubisch, C., Schroeder, B.C., Friedrich, T., Lutjohann, B., El-Amraoui, A., Marlin, S., Petit, C., and Jentsch, T.J. (1999). KCNQ4, a novel potassium channel expressed in sensory outer hair cells, is mutated in dominant deafness. Cell 96, 437-446. Abstract Article

Kunkel, M.T., Johnstone, D., Thomas, J.H., and Salkoff, L. (2000). Mutants of a temperature-sensitive 2-P domain potassium channel. J. Neurosci. 20, 7517-7524. Abstract

Lehmann-Horn, F., and Jurkat-Rott, K. (1999). Voltage-gated ion channels and hereditary disease. Physiol. Rev. 79, 1317-1372. Abstract

Levin, J.Z., and Horvitz, H.R. (1992). The Caenorhabditis elegans unc-93 gene encodes a putative transmembrane protein that regulates muscle contraction. J. Cell Biol. 117, 143-155. Article

Levin and Horvitz (1993). Three New Classes of Mutations in the Caenorhabditis elegans Muscle Gene sup-9. Genetics 135, 53-70. Abstract

Littleton, J.T., and Ganetzky, B. (2000). Ion channels and synaptic organization: analysis of the Drosophila genome. Neuron 26, 35-43. Abstract Article

MacKinnon, R., and Doyle, D.A. (1997). Prokaryotes offer hope for potassium channel structural studies. Nat. Struct. Biol. 4, 877-879. Abstract Article

Milkman, R. (1994). An Escherichia coli homologue of eukaryotic potassium channel proteins. PNAS 91, 3510-3514. Abstract

Papazian, D.M., Schwarz, T.L., Tempel, B.L., Jan, Y.N., and Jan, L.Y. (1987). Cloning of genomic and complementary DNA from Shaker, a putative potassium channel gene from Drosophila. Science 237, 749-753. Abstract

Pearson, W.L., Dourado, M., Schreiber, M., Salkoff, L., and Nichols, C.G. (1999). Expression of a functional Kir4 family inward rectifier K+ channel from a gene cloned from mouse liver. J. Physiol. 514, 639-653. Abstract Article

Petersen, C.I., McFarland, T.R., Stepanovic, S.Z., Yang, P., Reiner, D.J., Hayashi, K., George, A.L., Roden, D.M., Thomas, J.H., and Balser, J.R. (2004). In vivo identification of genes that modify ether-a-go-go-related gene activity in Caenorhabditis elegans may also affect human cardiac arrhythmia. Proc. Natl. Acad. Sci. USA 101, 11773-11778. Abstract Article

Pongs, O., Kecskemethy, N., Muller, R., Krah-Jentgens, I., Baumann, A., Kiltz, H.H., Canal, I., Llamazares, S., and Ferrus, A. (1988). Shaker encodes a family of putative potassium channel proteins in the nervous system of Drosophila. EMBO J. 7, 1087-1096. Abstract

Raizen, D.M., and Avery, L. (1994). Electrical activity and behavior in the pharynx of Caenorhabditis elegans. Neuron 12, 483-495. Abstract Article 
Richmond, J.E., and Jorgensen, E.M. (1999). One GABA and two acetylcholine receptors function at the C. elegans neuromuscular junction. Nature Neurosci. 2, 791-797. Abstract Article

Reiner, D.J., Weinshenker, D., and Thomas, J.H. (1995). Analysis of dominant mutations affecting muscle excitation in Caenorhabditis elegans. Genetics 141, 961-976. Abstract

Reiner, D.J., Newton, E.M., Tian, H., and Thomas, J.H. (1999). Diverse behavioral defects caused by mutations in Caenorhabditis elegans unc-43 CaM kinase II. Nature 402, 199-203. Abstract Article

Rubin, G.M., Yandell, M.D., Wortman, J.R., Gabor Miklos, G.L., Nelson, C.R., Hariharan, I.K., Fortini, M.E., Li, P.W., Apweiler, R., Fleischmann, W., Cherry, J.M., Henikoff, S., Skupski, M.P., Misra, S., Ashburner, M., Birney, E., Boguski, M.S., Brody, T., Brokstein, P., Celniker, S.E., Chervitz, S.A., Coates, D., Cravchik, A., Gabrielian, A., Galle, R.F., Gelbart, W.M., George, R.A., Goldstein, L.S., Gong, F., Guan, P., Harris, N.L., Hay, B.A., Hoskins, R.A., Li, J., Li, Z., Hynes, R.O., Jones, S.J., Kuehl, P.M., Lemaitre, B., Littleton, J.T., Morrison, D.K., Mungall, C., O'Farrell, P.H., Pickeral, O.K., Shue, C., Vosshall, L.B., Zhang, J., Zhao, Q., Zheng, X.H., and Lewis, S. (2000). Comparative genomics of the eukaryotes. Science 287, 2204-2215. Abstract Article

Saimi, Y., Loukin, S.H., Zhou, X.L., Martinac, B., and Kung, C. (1999). Ion channels in microbes. Methods Enzymol. 294, 507-524.

Salkoff, L., Kunkel, M., Wang, Z., Butler, A., Nonet, M., and Wei, A. (1998). The impact of the C. elegans genome sequencing project on $\mathrm{K}^{+}$channel biology. In: Function Potassium Channels: Molecular Structure, and Diseases. Current Topics in Membranes, Academic Press, New York, Chapter 46, pp. 9-27.

Salkoff, L., Butler, A., Fawcett, G., Kunkel, M., McArdle, C., Paz-y-Mino, G., Walton, N., Yuan, A., Wang, Z.W., and Wei, A. (2001). Evolution tunes the excitability of individual neurons. Neuroscience 103(4), 853-859. Abstract Article

Sanguinetti, M.C., Jiang, C., Curran, M.E., and Keating, M.T. (1995). A mechanistic link between an inherited and an acquired cardiac arrhythmia: HERG encodes the IKr potassium channel. Cell 81, 299-307. Abstract Article

Santi, C.M., Yuan, A., Fawcett, G., Wang, Z.W., Butler, A., Nonet, M.L., Wei, A., Rojas, P., and Salkoff, L. (2003). Dissection of $\mathrm{K}+$ currents in Caenorhabditis elegans muscle cells by genetics and RNA interference. Proc. Natl. Acad. Sci. USA 100, 14391-14396. Abstract Article

Sentenac, H., Bonneaud, N., Minet, M., Lacrout, F., Salmon, J.M., Gaymard, F., and Grignon, C. (1992). Cloning and expression in yeast of a plant potassium ion transport system. Science 256, 663-665. Abstract

Shtonda, B., and Avery, L. (2005). CCA-1, EGL-19 and EXP-2 currents shape action potentials in the Caenorhabditis elegans pharynx. J. Exp. Biol. 208, 2177-2190. Abstract Article

Shyng, S., and Nichols, C.G. (1997). Octameric stoichiometry of the KATP channel complex. J. Gen. Physiol. 110, 655-664. Abstract Article

Silverman, S.K., Kofuji, P., Dougherty, D.A., Davidson, N., and Lester, H.A. (1996). A regenerative link in the ionic fluxes through the weaver potassium channel underlies the pathophysiology of the mutation. Proc. Natl. Acad. Sci. USA 93, 15429-15434. Abstract Article

Singh, N.A., Charlier, C., Stauffer, D., DuPont, B.R., Leach, R.J., Melis, R., Ronen, G.M., Bjerre, I., Quattlebaum, T., Murphy, J.V., et al. (1998). A novel potassium channel gene, KCNQ2, is mutated in an inherited epilepsy of newborns. Nat. Genet. 18, 25-29. Abstract Article

Singh, N.A., Westenskow, P., Charlier, C., Pappas, C., Leslie, J., Dillon, J., Anderson, V.E., Sanguinetti, M.C., and Leppert, M.F. (2003). KCNQ2 and KCNQ3 potassium channel genes in benign familial neonatal convulsions: expansion of the functional and mutation spectrum. Brain 126, 2726-2737. Abstract Article

Smith, P.L., Baukrowitz, T., and Yellen, G. (1996). The inward rectification mechanism of the HERG cardiac potassium channel. Nature 379, 833-836. Abstract Article 
Thomas, J.H. (1990). Genetic analysis of defecation in Caenorhabditis elegans. Genetics 124, 855-872. Abstract

Trent, C., Tsuing, N., and Horvits, H.R. (1983). Egg-laying defective mutants of the nematode Caenorhabditis elegans. Genetics 104, 619-647. Abstract

Trudeau, M.C., Warmke, J.W., Ganetzky, B., and Robertson, G.A. (1995). HERG, a human inward rectifier in the voltage-gated potassium channel family. Science 269, 92-95. Abstract

Wang, Z.W., Saifee, O., Nonet, M.L., and Salkoff, L. (2001). Slo-1 Potassium channels control quantal content of neurotransmitter release at the C. elegans neuromuscular junction. Neuron 32,867-881. Abstract Article

Warmke, J., Drysdale, R., and Ganetzky, B. (1991). A distinct potassium channel polypeptide encoded by the Drosophila eag locus. Science 252, 1560-1562. Abstract

Warmke, J., and Ganetzky, B. (1994). A family of potassium channel genes related to eag in Drosophila and mammals. PNAS 91, 3438-3442. Abstract

Wei, A., Butler, A., and Salkoff, L. (2005). KCNQ-like potassium channels in Caenorhabditis elegans: conserved properties and modulation. J. Biol. Chem. 280, 21337-21345. Abstract Article

Wei, A., Jegla, T., and Salkoff, L. (1996). Eight potassium channel families revealed by the C. elegans genome project. Neuropharmacology 35, 805-829. Abstract Article

Wei, A., Yuan, A., Fawcett, G., Butler, A., Davis, T., Xu, S.Y., and Salkoff, L. (2002). Efficient isolation of targeted Caenorhabditis elegans deletion strains using highly thermostable restriction endonucleases and PCR. Nucl. Acids. Res. 30, e110-e117. Abstract Article

Weinshenker, D., Wei, A., Salkoff, L., and Thomas, J. (1999). Block of an ether-a-go-go-like K+ channel by imipramine rescues egl-2 excitation defects in Caenorhabditis elegans. J. Neurosci. 19, 9831-9840. Abstract

Yuan, A., Dourado, M., Butler, A., Blanton, N., Wei, A., and Salkoff, L. (2000). Slo-2, A K+ channel with a unique C1- dependence. Nature Neurosci. 3, 771-779. Abstract Article

Yuan, A., Santi, C.M., Wei, A., Wang, Z.W., Pollak, K., Nonet, M., Kaczmarek, L., Crowder, C.M., and Salkoff, L. (2003). The sodium-activated potassium channel is encoded by a member of the Slo gene family. Neuron 37, 765-763. Abstract Article

Zhu, X.L., Vaillant, B., Loukin, S.H., Kung, C., and Saimi, Y. (1995). YKC1 encodes the depolarization-activated $\mathrm{K}+$ channel in the plasma membrane of yeast. FEBS Lett. 373, 170-176. Abstract Article

All WormBook content, except where otherwise noted, is licensed under a Creative Commons Attribution License 